\section{OAB - Patientenwünsche ernst nehmen}

— Die überaktive Blase („overactive bladder syndrom", OAB) ist eine multifaktorielle Erkrankung, daher erfordert sie eine individuelle Diagnose und Therapie. Welches Medikament für welchen Patienten das richtige ist, richtet sich überwiegend nach individuellen Patientenfaktoren wie Nebenwirkungen, Polypharmazie oder Komorbiditäten. Ein an den Wünschen des Patienten orientiertes therapeutisches Vorgehen ist dabei nach Einschätzung von Dr. Ruth Kirschner-Hermanns, Aachen unabdingbar.

Trospiumchlorid, ein quartäres Amin, hat sich in der OAB-Therapie bewährt: Die nur wenig lipophile Substanz überschreitet die Blut-Hirn-Schranke kaum, das Risiko für ZNSNebenwirkungen ist daher gering, erklärt Dr. Christian Gratzke, München. Die neue Retardformulierung (uriVesc ${ }^{\circledR}, 60 \mathrm{mg}$ ) für die einmal tägliche Einnahme erwies sich in zwei randomisierten, placebokontrollierten Phase-III-Studien mit 1.165 OAB-Patienten als wirksam und sicher [Staskin et al. 2007, Dmochowski et al. 2008]. Im Verumarm verbesserte sich die Symptomatik bereits nach den ersten Tagen. Im Verlauf der zwölfwöchigen Behandlungsphase reduzierte sich die tägliche Miktionsfrequenz nochmals deutlich, auch die Inkontinenzepisoden verringerten sich erneut.

Die Nebenwirkungsprofile der einzelnen Anticholinergika sind gerade beim älteren, oft multimorbiden Patienten mit Polymedikation klinisch bedeutsam, so Prof. Helmut Madersbacher, Innsbruck. Medikamenteninteraktionen sind selten. Eine aktuelle Studie zeigt, dass sich bei Patienten, die aufgrund einer Alzheimer-Demenz bereits Cholinesterasehemmer einnahmen, die kognitiven Funktionen unter Trospiumchlorid anders als unter Tolteridin nicht verschlechterten. Laut Madersbacher wird Trospiumchlorid in der PRISCUS-Liste als einzige medikamentöse Therapiealternative zu jenen Anticholinergika genannt, die als potenziell inadäquate Medikation für ältere Menschen eingestuft wurden. cm

Pressefrühstück uriVesc ${ }^{\circledR}$ „,Heterogene Patientenbilder, eine Lösung - Behandlungsfortschritte in der Therapie der überaktiven Blase“ im Rahmen des 62. Kongresses der Deutschen Gesellschaft für Urologie, Düsseldorf, 24. September 2010 Veranstalter: Rottapharm|Madaus, Köln

\title{
Zoledronat: Knochenschutz und antitumoröse Effekte
}

\begin{abstract}
- Das Aminobisphosphonat Zoledronat (Zometa ${ }^{\circledR}$ ) ist eine etablierte Therapie in der Prävention skelettbezogener Komplikationen bei Patienten mit fortgeschrittenen, auf den Knochen ausgedehnten Tumorerkrankungen. Zahlreiche klinische Studien belegen die knochenschützende Wirkung: Verglichen mit Placebo reduziert Zoledronat die skelettale Komplikationsrate von $22 \%$ (Prostatakarzinom) bis $65 \%$ (Nierenzellkarzinom). Aktuelle Arbeiten belegen nun auch einen antitumorösen Effekt des Bisphosphonats, berichtete Prof. Ulf Tunn, Offenbach.
\end{abstract}

Die AZURE-Studie zeigte bei Patientinnen mit Mammakarzinom, dass die neoadjuvante Kombination aus Zoledronat und Chemotherapie die Tumorgröße um $43 \%$ reduziert und die pathologische Komplettremission um $70 \%$ steigert. Für die adjuvante Situation wies die 48-Monats-Analyse der ZO-FAST-
Studie nach, dass die Kombination mit einer endokrinen Therapie beim postmenopausalen Mammakarzinom das krankheitsfreie Überleben um $41 \%$ verlängert -im Vergleich zur verzögerten Gabe, die erst bei Knochendichteverlust oder Fraktur einsetzte.

Beim ossär metastasierten Nierenzellkarzinom verlängerte Zoledronat das progressionsfreie Überleben um fast sechs Monate (256 Tage vs. 89 Tage unter Placebo). Auch das Gesamtüberleben wurde durch Zoledronat verlängert: Beim Harnblasenkarzinom mit Knochenmetastasen waren nach einem Jahr noch 36,3\% der Patienten am Leben, unter Placebo überlebte keiner. koc

Satellitensymposium „Behandlung urologischer Tumoren heute" im Rahmen des 62. Kongresses der Deutschen Gesellschaft für Urologie, Düsseldorf, 24. September 2010 Veranstalter: Novartis Oncology, Nürnberg

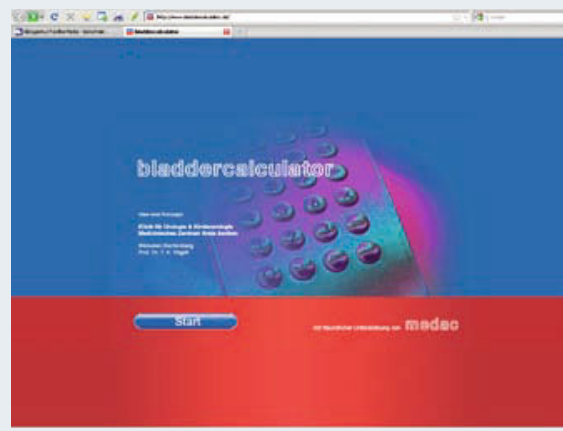

Der Bladdercalculator als App

Der „Bladdercalculator“, den es im World Wide Web unter der InternetAdresse www.bladdercalculator.de schon seit 2005 gibt, kann jetzt auch als iPhone App im App Store kostenlos heruntergeladen werden. Das Tool dient dazu, die Einteilungen von Patienten mit Harnblasenkarzinom in Risikogruppierungen zu vereinfachen und damit die Indikation zur intravesikalen Instillationstherapie zu optimieren. Dank des iPhone Apps lässt sich diese Berechnung jetzt auch unterwegs durchführen.

Nach Informationen von medac, Wedel

\section{Neue Broschüre für Nierenpatienten}

Mitte September ist der „Genzyme Nieren-Kompass" erschienen - eine Broschüre für Menschen mit chronischer Nierenerkrankung. Auf 34 Seiten finden Patienten hier für alle Stadien der Krankheit grundlegende Informationen und eine schnelle Orientierung. Inhalte der Broschüre sind unter anderem Ernährungstipps, Wissenswertes rund um die phosphatarme Ernährung und Informationen zum Erhalt der Gefäßgesundheit. Auch die Dialyseverfahren Hämodialyse und Peritonealdialyse werden vorgestellt. Ärzte können die Broschüre per E-Mail (niere-infode@genzyme.com,www.nierenund-gefaesse.de) per Telefon oder Fax bestellen (Tel.: $06102 / 3674-0$ oder Fax: -6 oo).

Nach Informationen von Genzyme, Neu-Isenburg 